ISBN 978-93-84468-92-7

International Conference on Issues in Education, Literature, Humanities and Social Sciences

(IELHSS-2017)

Kuala Lumpur (Malaysia) Jan. 4-5, 2017

\title{
Dialogue and Social Surfing: Different Communication Strategies in the Context of Subcultural Communities
}

\author{
Irina Simonova ${ }^{1}$ \\ ${ }^{1}$ Ural state pedagogical university (Dept. of Philosophy and Acmeology)/ Ural federal university named after \\ the first President of Russia B.N. Yeltsin (dept. of Social philosophy)
}

\begin{abstract}
This article analyzes the phenomenon of subcultural community in context of socio-topological methodology. We allocate three important aspects:

1) Intensive reflection and interest of participants in concrete activity in transforming the surrounding validity in borders of the life compatibility and effective transformation of social life make subcultures intersystem communities which have unique characteristics and actively represent it in the society. So, subcultural communities aspire through the work of body with concrete physical space at the time to seize and transform symbolical spaces of the society.

2) The most of subcultures are an important element of intercultural and intracultural dialogical mutual relations. They transport and put another cultural ways of the compatibility of various cultures into the life, and of through the application of alien practice and the body technique and their combination with local features. They also realize immediate intercultural interaction, and consequently they can be considered as a transcultural phenomenon. Mediality as one of the essential characteristics of a subculture expresses a world tendency to the intercultural dialogue. It shows a reality of its realization due to the potential of self-organizing of the human activity and the recognsing the value of the cultural variety stimulating the development of culture. Namely dialogical communicative strategy is the dbase of the subculture with its efficient character.

3) We postulate the conception of social surfing as communicative strategy and try to collate it with theory of communication in subculture. Social surfing is the communicative strategy projecting the special logic of compatibility in social process: it is an endless fast traffic from community to community, which is accompanied by a corresponding change in the system action and communication. This approach to social relations forms a special type of integration which will never be quite complete. It's always temporary and only imitates the fullness of social relationships. Choosing the social surfing, the actor just alternately uses the techniques and methods of communication. He never acts as a Creator of social reality, and he is only the consumer. That strategy is characteristic of modern youth including some members of the subculture but it doesn't create the conditions of the dialogical relations and it's inefficient.
\end{abstract}

Keywords: subculture, community, dialogue, social surfing, communication strategy, social topology

\section{Introduction}

The world globalization processes which intensively occurring last decades are provoke considerable shifts in organization of the space, which are characterized by transition from the local geographical place to union symbolical space, it's on the one hand, expands borders of communications, but on the other it's pushes together the world community in the union symbolical territory.

The situation becomes a fertile field for conflicts «Pernicious from the point of view of respect human advantage and public welfare» [1, p. 14], arising at different levels of social being. In this situation process of settlement already arisen oppositions is appear the extremely difficult. 
And so «segregated and closed for each other communities generate a climate, which is hostile for an individual autonomy of the person, interfere with realization of human rights and the basic freedoms» $[1, \mathrm{p} .16]$ also it generate dangerous intensity of society, to overcome which sometimes practically not probably, what, finally deprives of all «benefits from the new cultural opening which is necessary for development of the person and a society in the globalized world» [4, p. 14].In this reason formation the strategy prevention of cultural and sociality oppositions which would guarantee preservation originality of each participants sees priority, but it's allowed to coexist it in the uniform symbolical space of the present-day world. In particular, it is a question of the situation connected with a phenomenon of cultural variety, which is become the obvious and integral characteristic of the currently globalized world.

According to materials of the «White paper» of the Council of Europe on intercultural dialogue (Strasbourg, 2008), the dialogue - is the way to which we should go [1, p. 9] to satisfy requirements of present societies in overcoming by the conflicts connected with increase of cultural distinctions. In the same place «dialogue is understood as the open and valid exchange of opinions on the basis of mutual understanding and respect between different people, and also groups people of the various ethnic, cultural, religious and language accessory which are having different historical roots» [1,p. 10]. So, the head of public thought towards acceptance in cultural variety through system of cultures dialogue and representation formation about the personal role and the responsibility of the person for a condition to allcommunity appears a priority direction. But appeal of this approach is obvious in the same degree in what the complexities connected with its concrete realization in the conditions of a society because main difficult is the valid absence of clearness concerning practical value concept of the intercultural dialogue [1, p. 9].We assert that studying the communication mechanism of subcultural communities can become a way to clearing of this question.

At the same time, we need to distinguish productive dialogic relations of subcultural communities and the phenomenon of social surfing as unproductive communicative strategy that is not conducive to dialogic relations. Both communicative strategies are widely used by modern youth; they both are determined by specific mobile polymorphic and open condition of the social space. Both may be used by the party in subculture community context. But they are of different nature so they must be divided at the theory level for the further effective comprehension of the social reality. However, we should find what place and what role they play, and the main thing is what is the mechanism of their participation in global dialogical process of a society. So, analyzing activity of subcultural communities in a context of dialogical relations in a society will allow us:

- to consider mechanisms of the realization by social dialogue through estimation of ways functioning subcultures, as communities designed and realized by the participants

- to demonstrate orientation activity of these communities to concrete activity on transformation of the surrounding validity towards a dialogue installation/ breaking in borders of available compatibility and behind its limits;

- to be assured of necessity and possibility of dialogical relations thanks to cultivation of internal potential a society as they visualize a situation of cultural variety and a tendency of communities to construction social dialogical communications;

- to point out the essential differences between the productive communicative strategy in subcultural communities, and counter-productive strategy of social surfing.

This course will allow to actualize a problematic of dialogue and social surfing within the limits of a phenomenon of subcultural communities to estimate its importance in borders present social processes. We recede from operating by concepts from area of abstract systems, from culture and subculture competition as certain conditional programs, but we address to concrete social communications in their real existential measurement.

\section{Methodology}

Such consideration at a studied phenomenon allows me to move research to prospect of socially-topological analysis, it could helps us comprehending society without being based on parcels of philosophy of consciousness, and, at the same time, avoiding with the errors connected absolutization of social structure. [2, p. 6] Such approach has provided complete perception of it complex the social phenomenon localized within the concrete space, and as a result direct communications of people. Sociality will be taken by us not as what "already is", and as the implementation of joint activities of people and the arising of a certain connection 
between them [3]. Strategy thus can be considered d the context of the live social localities. People are working together and communicating in mobile communities of creators and bearers of a unique and discrete social practices and techniques, creating special places of sociality and communicating in specific ways. In the global information environment young person strives to feel a part of many social and cultural modifications to be "everywhere". Instead of traditional organizational forms, subculture, do not impose strict obligations and do not have a rigid structure: they are discrete, polymorphic, affordable and always incomplete.

Reconstructing a phenomenon of subcultural communities in marked by us «system of coordinates» we also can regard it as carriers realizing and broadcasting a combination of some original topogical construction, we allocate with its complex specific social the technician and an expert, providing cooperation and accommodation of agents, etc. drawing up by them special «places» of the sociality - toposes. Concept «topos», expressing uniqueness of existence of any social organism, in our case of subculture, «specifies in special type of relations inherent in it, structure, character of reproduction and updating the developed order» [4, p. 9]. So, along with the traditional «places» there is a creation of the new «places» a sociality, accommodation in which through interaction of a corporality and district builds system of existence subcultural communities, forming them as original set topologems as unique sociocultural formation realized in a concrete sociality.

\section{Results}

Speaking about subcultural communities language of social topology, the mechanism of their formation and functioning is described as special type of compatibility, a self-organizing special existential complex, is active represents in a society body through realization unique the technician, and designing of special places of a sociality, and by that its (society) the transforming. Considering subcultural communities as the constructive phenomenon projecting the special logic of compatibility in social process it is important to notice that the intensive reflection, interest of participants in concrete activity on transformation of the surrounding validity to borders of real compatibility and effective transformations of real social life do subculture by the complete system realized at all levels of a sociality as difficult intersystem community, possessing unique essential structurally functional, axiological and teleological characteristics and is active represents othe specified forms in society space It is important, what this social space is built by communities meaningly and practically during coexistence, in the course of the organized, ordered activity defined collectivity, and because «the person isn't simply shipped, but it actively is - that is cooperate itself together with others.» [4, p. 45]

It specifies in a special role of human activity when due to atomic desire and joint activity which isn't supported by additional motivation, except own desire in some measure differently to reproduce with-life in borders of the district so that the future had character distinct from the past. An essence it's becoming in the space and becoming in the time. Thus, we can speak about human activity of actualization and a private responsibility going from internal resources for a condition of the surrounding validity.

Activity of subcultural communities arises on breaks of social processes, on those sites which appear in the field of the most significant public problems, marked (allocated) as well a society as a whole. Such coincidence of marking «problem» And the significant zones of public practice which are carried out by a society and subcultural communities, specifies in their indissoluble remedial interrelation. Conditions for self-organizing of subcultural communities are put, naturally, in the mechanism of reproduction of a sociality. Similar vision with reference to subcultural process allows reconsidering and overcoming its settled artificial opposition cultural, and specifies that they are carried out more likely as the different parties of uniform process and are in certain dialogue.

It is important that subcultural communities marks necessity of social dialogue on a rank of the major questions from area of ecology, the right, economy and a wide spectrum of social problems. Thus they realize a considerable quantity unique social an expert by means of which they not only show necessity of reconsideration those or other questions, but for direct active action, that is for work as direct space and the corporality, form alternative ways of the decision problems in the local district.

It does not matter whether the subculture support positive values such as tolerance and openness, technically it always stand on a position of active development and maintenance of dialogical strategy, since it is based on communication, involvement of new members, networking with communities of like-minded people, including those who live in the context of other cultural conditions. 
In that case, if we look at subcultural communities, we can see that the majority of them appear direct participants of social and cultural dialogical mutual relations. This phenomenon should be considered as result of the self-organized dialogue in three basic directions [5].

The first direction - it is dialogical relations «Institutional community - Subcultural community». Is the most obvious. Double position of subcultural communities as the original included autonomy allows them to create and broadcast special alternative senses and to be reproduced thereby in all uniqueness, forming and developing new topologic compatibility, belonging and remaining, at the same time, a part of that social system in which it arises also which locally transforms thanks to designing new topological constructions, providing a projection of the reconstructed «symbolical universum», understood here as a complex of social representations, knowledge, the purposes, motive forces and etc., drawing the uniform world for the given social generality. Thanks to the described tendency also there is a process of updating and culture enrichment. Though the exchange of the cultural information occurs constantly, participants continue to keep the status of an original autonomy. Thus, it is possible to draw a conclusion on the real dialogue occurring between a society and subcultural communities. And shows internal requirement for such self-organizing as feels real necessity for updating.

The second direction of dialogue which is important to consider it is «Subcultural community A Subcultural community B». Social dialogue between various subcultural communities plays an important role for their formation and is the integral process for their growth. We can fix constant migrations communicative and corporal the technician between various communities, and also tendencies to formation similar thoposes that, finally, leads to development of conterminous or similar ways of compatibility. Actually, many communities appear carriers similar topologems, it appears result of dialogical relations. Activity of communities is quite often directed on similar zones of the validity that leads to similar activity on its transformation. From here and similar modes of work with a body, its registration, conterminous physical places and their symbolical weight. It complexity of clear split of some communities among themselves also speaks, distinctions can sometimes be very insignificant. It is necessary to note, orientation to dialogue becomes key self-organizing for ways of subcultural communities. Even in case of animosities between some of them, dialogical relations are built as though by contradiction, that is the community directs the activity according to topologems hostile community.

The third dialogical line « Institutional community A - subcultural community - Institutional community B» represents for us the greatest interest Overwhelming majority of subcultures don't belong to the concrete country and, having arisen in certain territory, have development on perfect other territories, being reconstructed taking into account their special lines, however keeping the primary central idea due to which they can be fixed worldwide as something uniform (Fig 1.)

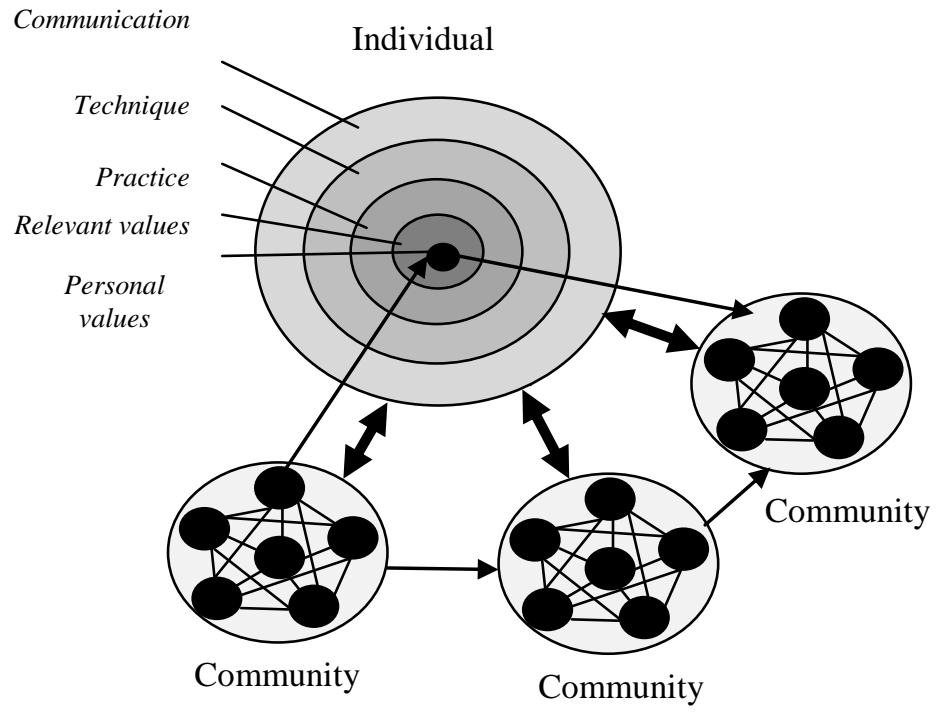

Fig. 1: Individual in dialogical strategy

Representing a common cultural phenomenon, they transport and enter into life of various cultures anotherculturel topologems and through application alien an expert and the technician of a body and 
communication, and their combination with local topologems realize direct intercultural interaction. Considerable ethnical and folklore the appeal to various cultural traditions (including reactualisation by archaic the various countries) in use of a body and the technician of compatibility, such as the suit, a tattoo, piercing, ritual, dance, language, etc., allows to do a conclusion about subculture as about a transcultural phenomenon, as a matter of fact, and representing cultural dialogue that is important, proceeding not from political, religious or other institutes, and from people in their direct combination. Thus, we can fix concept of subculture as result of the self-organized dialogue of cultures. Mediality as one of essential characteristics of subculture expresses a world, general tendency to intercultural dialogue and ascertains a reality of realization of the last, thanks to self-organizing of human activity and a recognition of value of cultural variety, as, undoubtedly, stimulating culture development.

The social surfing is absolutely different communicative strategy. In actual conditions the classical social institutes in some way begin to loose their influence, the individual stops to be strongly linked to the traditional alliances like family, confession and nation. The adherence with a specific territory (the region, the city, the country) [6] is also disappearing. In global informative environment the young person aspires to feel himself as a part of many social and cultural modification, to be "everywhere" [7]. Modern alliances are characterized by the lack of the hard relations: they are discrete, polymorphic, accessible and always unaccomplished. For the young people numerous subcultures now provide the possibility to transfer from one alliance to the other, the leap between the different communication flows, the shuffling and mastering of many cultural practices.

Now It comes not necessarily about some hard adherences, some participant's obligations, some serious transformations of the communicant's basic actions: in the long procession of transitions from one social locations to the other the human being can be easily become their part learning only the most obvious processes of the practices, contriving the fleeting game in "somebody", self-presenting in conditional membership. That social surfing, the quick and superficial movement between the real systems of the action and communication, represents special communicative tactic, some special type of the social involvement which never be full enough in usual way but just tends to imitate this fullness (Fig. 2)

The person in the process of social surfing is not productive, it is only the consumer. In the transitions from one community to another nothing will be produced, nothing will upgrade, nothing will be marked. During the transition from one community to another a person will be transport nothing, the cultural dialogue will not happen. Also some new values, technology, means of communication will not processed. A person will not do the transplant techniques and values from one community to another, because the surfer is moving from one community to another completely severs contact with it.

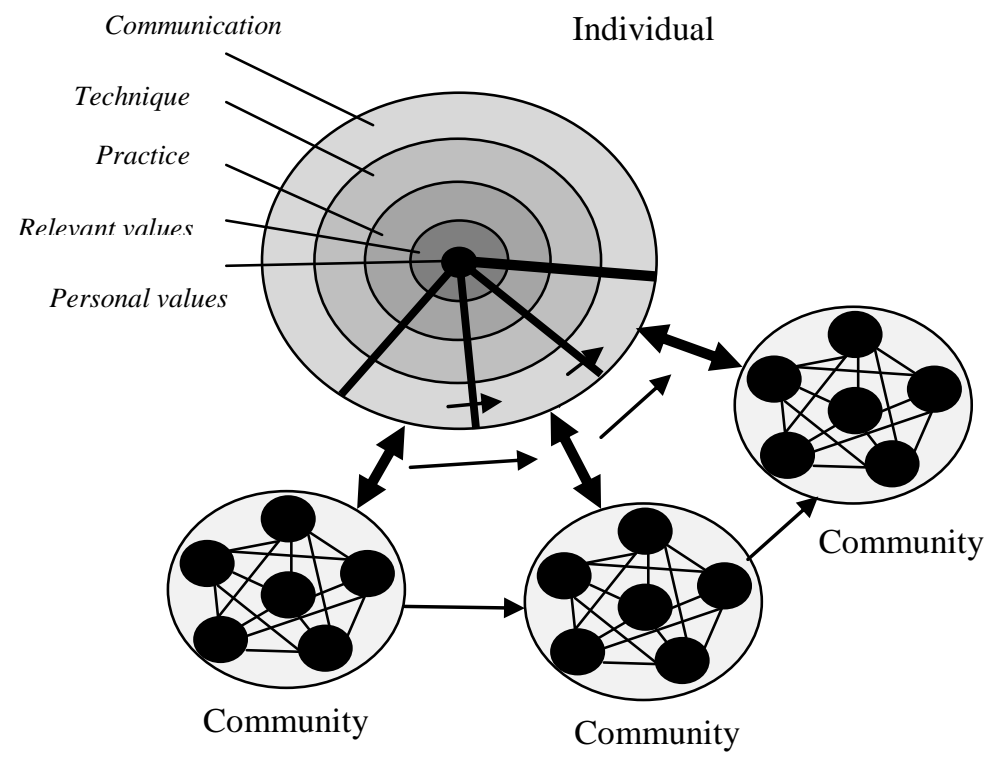

Fig. 2: Individual in social surfing strategy (in dynamic) 


\section{Conclusions}

Being special transcultural way of the organization of compatibility, subculture possesses powerful dialogical potential in borders of societies of modern type. As a unique social phenomenon, it not only personifies an actual condition of the globalized world, but also testifies to real advantage of intercultural dialogue. We want to pay attention to the fact that it is unacceptable to mix the phenomenon of social surfing (when the individual moves between communities and uses ready-made forms and technology in it) and the mobility of individuals in dialogic communication in subcultural communities (when the individual together with other creating new forms of sociality that provides it enrichment).

\section{Acknowledgements}

The study was supported by the Russian Foundation for Basis Research "Social surfing: Foresight civil subjectivity of the Russian youth" project 16-33-01057;

We are grateful to Alexey Lushanov for help in the translation of the text of the article.

\section{References}

[1] White Paper on Intercultural Dialogue. Living together in equal dignity. Strasbourg, 2008

[2] S.A. Azarenko Community of body, Moscow, Russia: Academ. Pr. M. 2007

[3] J. Law "After ant: Topology, Naming and Complexity" in Actor-Network and After, ed. by J. Law, J. Hassard. Oxford, 1999. - p.p. 3-14.

[4] S.A. Azarenko Topology of cultural reproduction, Ekaterinburg, Russia: Ur.university, 2000

[5] S. H. Schwartz Universals in the content and structure of values: Theory and empirical tests in 20 countries, Advances in experimental social psychology. New York: Academic Press, 1992. pp. 1-65

[6] I.A. Simonova, "From the Cultural Diversity to the Intercultural Dialogue: Sociological Topology of Subcultural Communities", Education and science, № 10, p.p. 88 - 99, 2010.

[7] I.A. Simonova, O.V. Kruzhkova, I.V. Vorobyeva, "Social surfing: specific value orientations of youth in contemporary society,” Pedagogical education in Russia, № 5, p.p. 43 - 48, June 2015. 